\title{
Self-gravitating spherically symmetric solutions in scalar-torsion theories
}

\author{
Georgios Kofinas, ${ }^{1, *}$ Eleftherios Papantonopoulos, ${ }^{2,3, \dagger}$ and Emmanuel N. Saridakis ${ }^{2,4, \$}$ \\ ${ }^{1}$ Research Group of Geometry, Dynamical Systems and Cosmology, \\ Department of Information and Communication Systems Engineering, \\ University of the Aegean, Karlovassi 83200 Samos, Greece \\ ${ }^{2}$ Physics Division, National Technical University of Athens, 15780 Zografou Campus, Athens, Greece \\ ${ }^{3}$ CERN-Theory Division, CH-1211 Geneva 23, Switzerland \\ ${ }^{4}$ Instituto de Física, Pontificia Universidad de Católica de Valparaíso, Casilla 4950 Valparaíso, Chile
}

(Received 27 February 2015; published 27 May 2015)

\begin{abstract}
We study spherically symmetric solutions in scalar-torsion gravity theories in which a scalar field is coupled to torsion with a derivative coupling. We obtain the general field equations from which we extract a decoupled master equation, the solution of which leads to the specification of all other unknown functions. We first obtain an exact solution which represents a new wormholelike solution dressed with a regular scalar field. Then, we find large distance linearized spherically symmetric solutions in which the space asymptotically is anti-de Sitter.
\end{abstract}

DOI: $10.1103 /$ PhysRevD.91.104034

PACS numbers: 04.50.Kd

\section{INTRODUCTION}

One of the main concerns in constructing a physically acceptable field theory is to avoid unphysical propagating modes, like ghosts. General relativity (GR) is one example of a healthy classical theory of gravity which is based on a unique spacetime metric along with its Levi-Civita connection, and it is constructed out of second derivatives in the metric tensor. The simple form of Hilbert-Einstein action leads to field equations with no more than second derivatives, resulting in a gravitational theory without ghosts [1], which, moreover, passes successfully with high accuracy all local observational tests both for weak and strong gravity [2].

Torsion is an important tensor besides curvature, which can be used for the construction of gravitational theories. In this case, one can use as dynamical variables the vielbein and the connection, and the field equations arise by variation with respect to these two variables. Soon it was realized that imposing the teleparallelism condition, it is possible to express equivalently the standard Einstein gravity [3-10] (or even Gauss-Bonnet gravity [11]) by a different geometry described in terms of torsion. This can be achieved by employing instead of the Levi-Civita connection the Weitzenböck one, which has vanishing curvature but a nonvanishing antisymmetric piece and

\footnotetext{
*gkofinas@aegean.gr

lpapa@central.ntua.gr

†Emmanuel_Saridakis@baylor.edu
}

Published by the American Physical Society under the terms of the Creative Commons Attribution 3.0 License. Further distribution of this work must maintain attribution to the author $(s)$ and the published article's title, journal citation, and DOI. therefore nonvanishing torsion, and thus the only dynamical variable that remains is the vielbein.

In a more general formulation of teleparallelism, an arbitrary connection of vanishing curvature is assumed, and everything is again expressed in terms of the torsion, but now both the vielbein and the connection are dynamical. In this formulation, the theory is both Lorentz and diffeomorphism invariant, while the former formulation lacks local Lorentz invariance. In its teleparallel formulation, Einstein gravity action consists of the torsion scalar $T$, which is a specific combination of quadratic torsion scalars and contains up to first-order vielbein derivatives. Then, the variation of this action gives exactly the Einstein gravitational field equations, and for this reason the theory is termed teleparallel equivalent of general relativity (TEGR) [3-10].

One interesting question is what kind of theories can be produced in which the torsion can give sizable physical effects that can differentiate these theories from GR. One approach that was put forward, inspired from the $f(R)$ modifications of GR, is to start from TEGR instead of GR, and construct $f(T)$ extensions. This new class of gravitational modification does not coincide with $f(R)$ and proves to have interesting local black hole solutions [12-21], and it gives variable cosmological models [22-33].

Another approach to probe the effects of torsion to GR is to couple the torsion to scalar fields. Self-gravitating scalar fields were used to study the dynamics of local black hole solutions of Einstein equations. These studies had resulted in the well-known no-hair theorems $[34,35]$ and in ways to avoid them [36-40]. In cosmology the scalar fields have also been used to investigate the features of the early Universe [41-43]. Apart from the simple minimal coupling case, the scalar field may be coupled nonminimally with curvature, since the appearance of terms of the form $f(\phi) R$ 
is motivated by many reasons, such as the variability of the fundamental constants, the Kaluza-Klein compactification scheme, or the low-energy limit of superstring theory.

To capture the effects of curvature in the early stages of cosmological evolution or near the horizon of a black hole, couplings of scalar fields directly to curvature through terms of the form $R g^{\mu \nu} \partial_{\mu} \phi \partial_{\nu} \phi$ were studied. However, these derivative coupling terms lead to field equations containing higher than second derivatives [44], and therefore ghosts. To remedy this pathology one has to couple the scalar fields to the Einstein tensor [45] and this term $G^{\mu \nu} \partial_{\mu} \phi \partial_{\nu} \phi$ is part of the general Horndeski Lagrangian which is known to result [46] in a healthy theory with only second-order field equations. These theories have interesting cosmological implications [45,47-49] and they give also local black hole solutions [50-56].

In this work we will discuss first couplings of torsion to scalar fields of the form $f(\phi) T$. We will show that such coupling in four or higher dimensions, for a diagonal vielbein, does not give nontrivial spherically symmetric solutions. ${ }^{2}$ We will then introduce a derivative coupling of a scalar field to torsion of the form $T g^{\mu \nu} \partial_{\mu} \phi \partial_{\nu} \phi$. This coupling, contrary to its curvature counterpart $R g^{\mu \nu} \partial_{\mu} \phi \partial_{\nu} \phi$, has the advantage that it leads to secondorder equations of motion. We will show that such a coupling gives spherically symmetric solutions which have distinct features from their GR counterparts. Note that the above derivative coupling between torsion and the scalar field is not the only one that can be constructed, since other combinations of $\partial_{\mu} \phi \partial_{\nu} \phi$ with quadratic (of even higher) powers of torsion could appear.

Our work is organized as follows. In Sec. II, after a brief review of scalar-tensor theories, we set up our theory of a scalar field coupled to torsion with a derivative coupling and present the field equations of the gravity-scalar system. In Sec. III we consider a spherically symmetric ansatz for the metric and we reduce the coupled field equations to a decoupled master equation from which the solutions are obtained. In Sec. IV we discuss an exact wormholelike solution, while in Sec. V we discuss large distance solutions. Finally, Sec. VI is devoted to our conclusions.

\section{DERIVATIVE COUPLING OF A SCALAR FIELD TO TORSION}

In this section, after a brief review of scalar-tensor theories, and in particular of a scalar field minimally coupled to gravity, we will introduce torsion, and we will set up the gravity-scalar field equations describing the torsion field coupled with a derivative coupling to a scalar field.

\footnotetext{
${ }^{1}$ The cosmological implications of these types of couplings were studied in [57-61].

${ }^{2}$ On the contrary, in three dimensions spherically symmetric solutions were found [62].
}

In $D$ dimensions the Einstein-Hilbert action with a scalar field minimally coupled to gravity is given by

$$
\begin{aligned}
S_{\min }= & \frac{1}{2 \kappa_{D}^{2}} \int d^{D} x \sqrt{|g|} \bar{R} \\
& -\int d^{D} x \sqrt{|g|}\left(\frac{1}{2} g^{\mu \nu} \partial_{\mu} \phi \partial_{\nu} \phi+V\right) .
\end{aligned}
$$

The Ricci scalar $\bar{R}$ is constructed from the Christoffel connection $\Gamma_{\mu \nu}^{\lambda}$. Indices $a, b, \ldots$ will refer to tangent space, while $\mu, \nu, \ldots$ are coordinate ones. A cosmological constant $\Lambda= \pm 3 l^{-2}$, where $l$ is the length of the (anti-)de Sitter [(A) dS] space, has been incorporated in the potential, so the total potential is $V(\phi)=\frac{\Lambda}{\kappa_{D}^{2}}+V_{1}(\phi)$. In four dimensions and for a negative cosmological constant this theory has a local black hole solution known as the Martinez-TroncosoZanelli (MTZ) solution [36] given by

$$
d s^{2}=B(r)\left[-F(r) d t^{2}+\frac{1}{F(r)} d r^{2}+r^{2} d \sigma^{2}\right],
$$

where

$B(r)=\frac{r\left(r+2 G_{N} \mu\right)}{\left(r+G_{N} \mu\right)^{2}}, \quad F(r)=\frac{r^{2}}{l^{2}}-\left(1+\frac{G_{N} \mu}{r}\right)^{2}$.

The constant $\mu$ can be identified with the mass of the black hole and $\kappa_{4}{ }^{2}=8 \pi G_{N}$, with $G_{N}$ the Newton's constant. The line element $d \sigma^{2}$ refers to a two-dimensional manifold of negative constant curvature. The scalar field is given by

$$
\phi=\sqrt{\frac{3}{4 \pi G_{N}}} \operatorname{arctanh}\left(\frac{G_{N} \mu}{r+G_{N} \mu}\right),
$$

and the potential $V_{1}$ is found to be

$$
V_{1}(\phi)=-\frac{3}{4 \pi G_{N} l^{2}} \sinh ^{2}\left(\sqrt{\frac{4 \pi G_{N}}{3}} \phi\right) .
$$

This is the simplest known hairy black hole solution of a scalar field minimally coupled to the curvature, which goes to zero at infinity and is regular on the horizon. Here we should stress an important property of this solution. If we switch off the scalar field we cannot get a black hole solution with fixed mass. In fact, there is only one integration constant $\mu$, and for $\phi \rightarrow 0$ the geometry approaches a massless black hole. This means that for a given mass there are two branches of different black hole solutions, the one with a nontrivial scalar field given by (2.2), and the vacuum solution (with $\phi=0$ ).

In writing (2.1) we used the usual Christoffel connection which by construction has zero torsion, and thus, all the gravitational information is incorporated by the curvature 
(Riemann) tensor. In the teleparallel formulation an alternative connection $\omega_{b c}^{a}$ is adopted, and the basic object is the torsion

$$
T_{b c}^{a}=\omega_{c b}^{a}-\omega_{b c}^{a}-e_{b}^{\mu} e_{c}^{\nu}\left(e^{a}{ }_{\mu, \nu}-e_{\nu, \mu}^{a}\right),
$$

expressed in terms of the arbitrary vielbein $e_{a}=e_{a}{ }^{\mu} \partial_{\mu}$ (with dual $e^{a}=e^{a}{ }_{\mu} d x^{\mu}$ ) and the connection $\omega_{b}^{a}=\omega_{b \mu}^{a} d x^{\mu}=\omega_{b c}^{a} e^{c}$. We mention that the torsion as defined in (2.6) is a tensor under local Lorentz transformations and under diffeomorphisms. The vielbein is assumed to be orthonormal, which implies that the metric is given by

$$
g_{\mu \nu}=\eta_{a b} e^{a}{ }_{\mu} e_{\nu}^{b},
$$

where $\eta_{a b}=\operatorname{diag}(-1,1, \ldots 1)$ is the Minkowski metric. The metric is assumed to be compatible with the connection $\omega_{b c}^{a}$, namely $\eta_{a b \mid c}=0$, and thus $\omega_{a b c}=-\omega_{b a c}$, where | denotes covariant differentiation with respect to $\omega_{b c}^{a}$. The teleparallelism condition is imposed by assuming that the curvature of $\omega_{b c}^{a}$ is zero, i.e. $R_{b \mu \nu}^{a}=\omega_{b \nu, \mu}^{a}-\omega_{b \mu, \nu}^{a}+$ $\omega^{a}{ }_{c \mu} \omega_{b \nu}^{c}-\omega^{a}{ }_{c \nu} \omega^{c}{ }_{b \mu}=0$ (note however that the curvature $\bar{R}^{a}{ }_{b \mu \nu}$ constructed from the Christoffel connection is not zero). One way to realize this condition is by considering the Weitzenböck connection, which is defined in terms of the vielbein in all coordinate frames as $\omega^{\lambda}{ }_{\mu \nu}=e_{a}{ }^{\lambda} e^{a}{ }_{\mu, \nu}$, and therefore $\omega_{b c}^{a}=0$ in the preferred frame $e_{a}$ that defines the connection.

Then, the following torsion scalar can be defined as

$$
\begin{aligned}
T & =\frac{1}{4} T^{\mu \nu \lambda} T_{\mu \nu \lambda}+\frac{1}{2} T^{\mu \nu \lambda} T_{\lambda \nu \mu}-T_{\nu}{ }^{\nu \mu} T_{\lambda \mu}^{\lambda} \\
& =S^{\mu \nu \lambda} T_{\mu \nu \lambda},
\end{aligned}
$$

where the tensor $S^{\mu \nu \lambda}$ is defined by

$$
S^{\mu \nu \lambda}=\frac{1}{2} \mathcal{K}^{\nu \lambda \mu}+\frac{1}{2}\left(g^{\mu \lambda} T_{\rho}{ }^{\rho \nu}-g^{\mu \nu} T_{\rho}{ }^{\rho \lambda}\right)=-S^{\mu \lambda \nu}
$$

and

$\mathcal{K}_{\mu \nu \lambda}=\frac{1}{2}\left(T_{\lambda \mu \nu}-T_{\nu \lambda \mu}-T_{\mu \nu \lambda}\right)=-\mathcal{K}_{\nu \mu \lambda}=\omega_{\mu \nu \lambda}-\Gamma_{\mu \nu \lambda}$

is the contorsion tensor. In the teleparallel formulation, torsion is used to express the action (2.1) in an equivalent form (up to boundary terms) as

$S_{\min }=-\frac{1}{2 \kappa_{D}^{2}} \int d^{D} x e T-\int d^{D} x e\left(\frac{1}{2} g^{\mu \nu} \partial_{\mu} \phi \partial_{\nu} \phi+V\right)$.
Since $T$ differs from $\bar{R}$ by boundary terms, namely $T=$ $-\bar{R}+2 T_{\nu}{ }^{\nu \mu}{ }_{; \mu}$ with ; denoting covariant differentiation with respect to the Christoffel connection, the above action (2.12) indeed gives exactly the same equations with the action (2.1). For example, the hairy black hole solution (2.2), (2.4), (2.5) can be obtained from the action (2.12) in four dimensions.

In this work we are interested in finding local spherically symmetric solutions in which the presence of the torsion could leave an explicit signature on the solution, other than from the trivial one found from the action (2.12). To achieve this, the action (2.12) has to be modified in such a way as to include a coupling of torsion to the scalar field.

The easiest way to modify the action (2.12) is to add a direct coupling of the scalar field to torsion

$$
\begin{aligned}
S_{\text {non-min }}= & -\frac{1}{2 \kappa_{D}^{2}} \int d^{D} x e T \\
& -\int d^{D} x e\left(\frac{1}{2} g^{\mu \nu} \partial_{\mu} \phi \partial_{\nu} \phi+V+\xi f(\phi) T\right) .
\end{aligned}
$$

The resulting theory is different from its corresponding curvature counterpart and it leads to novel features when applied to a cosmological geometry [57-61]. However, it is not easy to apply the above theory to a spherically symmetric geometry in four dimensions. The reason is that for a diagonal vierbein the coupling of the scalar field to torsion gives a new $(r-\theta)$ equation which acts as a strong constrain to the coupled field equations. Thus, the arising geometry is trivial and does not give hairy spherically symmetric solutions. Note, that in three dimensions the absence of this off-diagonal field equation allows hairy black hole solutions to be found [62].

Another possible extension of the action (2.12) is to include a derivative coupling of the scalar field to torsion. In particular, we consider such a coupling with the torsion scalar $T$, namely

$$
\begin{aligned}
S= & -\frac{1}{2 \kappa_{D}^{2}} \int d^{D} x e T \\
& -\int d^{D} x e\left[\left(\frac{1}{2}-\xi T\right) g^{\mu \nu} \partial_{\mu} \phi \partial_{\nu} \phi+V\right] .
\end{aligned}
$$

The coupling parameter $\xi$ has dimensions of length square, and therefore, $\sqrt{|\xi|}$ introduces a new length scale in the theory.

In order to extract the field equations, we need to perform variation with respect to the vielbein $e_{a}{ }^{\mu}$ and the scalar field. Additionally, and more formally, in order to implement the teleparallelism condition $R_{a b c d}=0$, one adds in the action (2.14) the term $\lambda^{a b c d} R_{a b c d}$, where $\lambda^{a b c d}$ is a Lagrange multiplier. Thus, variation with respect to $\lambda^{\text {abcd }}$ 
naturally provides $R_{a b c d}=0$, which can be considered as the equation of motion for the connection $\omega_{b c}^{a}$. Variation of the action (2.14) with respect to the connection $\omega_{b c}^{a}$ gives the equation of motion for the Lagrange multiplier. Since

$$
\begin{aligned}
-\frac{1}{4} \delta_{e}\left(e T \phi_{, \mu} \phi^{\mu}\right)= & -e S^{d c a} \omega_{b d c} \phi_{, \rho} \phi^{, \rho} e^{b}{ }_{\mu} \delta e_{a}{ }^{\mu}-\left(e S_{a}{ }^{\mu \nu} \phi_{, \kappa} \phi^{, \kappa} \delta e^{a}{ }_{\nu}\right)_{, \mu}+\left\{\left[\left(e S_{\kappa}^{\lambda \nu} e_{b}{ }^{\kappa}\right)_{, \nu} e^{b}{ }_{\mu}+e\left(\frac{1}{4} T \delta_{\mu}^{\lambda}-S^{\nu \kappa \lambda} T_{\nu \kappa \mu}\right)\right] \phi_{, \rho} \phi^{, \rho}\right. \\
& \left.-\frac{1}{2} e T \phi_{, \mu} \phi^{, \lambda}-e S_{\mu}{ }^{\nu \lambda}\left(\phi_{, \kappa} \phi^{, \kappa}\right)_{, \nu}\right\} e^{a}{ }_{\lambda} \delta e_{a}{ }^{\mu}
\end{aligned}
$$

it arises that

$$
\begin{aligned}
\delta_{e} S= & -\int d^{D} x\left(\frac{2}{\kappa_{D}^{2}}-4 \xi \phi_{, \rho} \phi^{, \rho}\right) e S^{d c a} \omega_{b d c} e^{b}{ }_{\mu} \delta e_{a}{ }^{\mu}-\int d^{D} x\left[\left(\frac{2}{\kappa_{D}^{2}}-4 \xi \phi_{, \rho} \phi^{, \rho}\right) e S_{a}{ }^{\mu \nu} \delta e^{a}{ }_{\nu}\right]_{, \mu} \\
& +\int d^{D} x\left\{\left(\frac{2}{\kappa_{D}^{2}}-4 \xi \phi_{, \rho} \phi^{, \rho}\right)\left[\left(e S_{\kappa}^{\lambda \nu} e_{b}{ }^{\kappa}\right)_{, \nu} e^{b}{ }_{\mu}+e\left(\frac{1}{4} T \delta_{\mu}^{\lambda}-S^{\nu \kappa \lambda} T_{\nu \kappa \mu}\right)\right]\right. \\
& \left.+4 \xi\left[\frac{1}{2} e T \phi_{, \mu} \phi^{, \lambda}+e S_{\mu}{ }^{\nu \lambda}\left(\phi_{, \kappa} \phi^{, \kappa}\right)_{, \nu}\right]+e\left(\frac{1}{2} \phi_{, \rho} \phi^{, \rho} \delta_{\mu}^{\lambda}-\phi_{, \mu} \phi^{, \lambda}+V \delta_{\mu}^{\lambda}\right)\right\} e^{a}{ }_{\lambda} \delta e_{a}{ }^{\mu},
\end{aligned}
$$

where $\phi^{\mu}=g^{\mu \nu} \phi_{, \nu}$. Ignoring the boundary term and setting $\delta_{e} S=0$, we obtain

$$
\begin{aligned}
& \left(\frac{2}{\kappa_{D}^{2}}-4 \xi \phi_{, \rho} \phi^{, \rho}\right)\left[\left(e S_{\kappa}^{\lambda \nu} e_{b}{ }^{\kappa}\right)_{, \nu} e^{b}{ }_{\mu}+e\left(\frac{1}{4} T \delta_{\mu}^{\lambda}-S^{\nu \kappa \lambda} T_{\nu \kappa \mu}\right)\right]+4 \xi\left[\frac{1}{2} e T \phi_{, \mu} \phi^{, \lambda}+e S_{\mu}{ }^{\nu \lambda}\left(\phi_{, \kappa} \phi^{, \kappa}\right)_{, \nu}\right] \\
& +e\left(\frac{1}{2} \phi_{, \rho} \phi^{\rho} \delta_{\mu}^{\lambda}-\phi_{, \mu} \phi^{, \lambda}+V \delta_{\mu}^{\lambda}\right)-\left(\frac{2}{\kappa_{D}^{2}}-4 \xi \phi_{, \rho} \phi^{, \rho}\right) e S^{d c a} \omega_{b d c} e_{a}^{\lambda} e^{b}{ }_{\mu}=0 .
\end{aligned}
$$

We mention that the above expressions contain also the connection $\omega_{a b c}$. Assuming for simplicity the Weitzenböck connection, that is setting $\omega_{a b c}=0$, which is a solution of the equation of motion $R_{a b c d}\left(\omega^{a}{ }_{b c}\right)=0$, we acquire the field equations containing only the vielbein, namely

$$
\begin{aligned}
& \left(\frac{2}{\kappa_{D}^{2}}-4 \xi \phi_{, \rho} \phi^{, \rho}\right)\left[\left(e S_{\kappa}^{\lambda \nu} e_{b}{ }^{\kappa}\right)_{, \nu} e^{b}{ }_{\mu}+e\left(\frac{1}{4} T \delta_{\mu}^{\lambda}-S^{\nu \kappa \lambda} T_{\nu \kappa \mu}\right)\right]+4 \xi\left[\frac{1}{2} e T \phi_{, \mu} \phi^{, \lambda}+e S_{\mu}{ }^{\nu \lambda}\left(\phi_{, \kappa} \phi^{, \kappa}\right)_{, \nu}\right] \\
& +e\left(\frac{1}{2} \phi_{, \rho} \phi^{\rho} \delta_{\mu}^{\lambda}-\phi_{, \mu} \phi^{, \lambda}+V \delta_{\mu}^{\lambda}\right)=0 .
\end{aligned}
$$

Additionally, variation of the action (2.14) with respect to the scalar field $\phi$ gives

$$
\delta_{\phi} S=\int d^{D} x\left\{\left[e(1-2 \xi T) \phi^{\mu}\right]_{, \mu}-e \frac{d V}{d \phi}\right\} \delta \phi-\int d^{D} x\left[e(1-2 \xi T) \phi^{, \mu} \delta \phi\right]_{, \mu}
$$

from where the scalar field equation of motion is obtained as

$$
\left[e(1-2 \xi T) \phi^{\mu}\right]_{, \mu}-e \frac{d V}{d \phi}=0 .
$$

Summarizing, equations (2.18) are the gravity field equations, while equation (2.20) is the Klein-Gordon one for the scalar field. Observe that $T$ being a scalar can be identified with the Hubble parameter in a cosmological Friedmann-Robertson-Walker (FRW) background as $T=G_{00}=6 H^{2}$. This implies that the derivative coupling $T g^{\mu \nu} \partial_{\mu} \phi \partial_{\nu} \phi$ on a FRW background will give the same cosmological evolution as the derivative coupling of the scalar field to Einstein tensor $G^{\mu \nu} \partial_{\mu} \phi \partial_{\nu} \phi$. However, general cosmological perturbations will differentiate the two models because they will excite also the spatial components of the Einstein tensor.

In the next section we will study local spherically symmetric solutions of the field equations (2.18) and (2.20) and we expect to find solutions with features different than the local black hole solutions of theories with a scalar field coupled to Einstein tensor with a derivative coupling [50-56].

\section{SPHERICALLY SYMMETRIC SOLUTIONS}

In this section we look for spherically symmetric solutions of the theory with a derivative coupling of the torsion to the scalar field in four dimensions. We consider the general spherically symmetric metric ansatz of the form 


$$
d s^{2}=-N(r)^{2} d t^{2}+K(r)^{-2} d r^{2}+R(r)^{2} d \Omega^{2},
$$

where $d \Omega^{2}=d \theta^{2}+\sin ^{2} \theta d \varphi^{2}$ is the two-dimensional sphere, and $N(r), K(r)$ and $R(r)$ are three unknown functions. Note that we do not impose the relation $N(r)=$ $K(r)$ keeping the metric ansatz as general as possible. We could have chosen the gauge with $R(r)=r$, but we find it more convenient not to impose a particular gauge and to let also the function $R(r)$ be free. We consider the following diagonal vierbein which gives rise to the above metric:

$$
e^{a}{ }_{\mu}=\operatorname{diag}\left(N(r), K(r)^{-1}, R(r), R(r) \sin \theta\right),
$$

which is adequate to capture the main features of our theory.

Inserting the vierbein (3.2) into the definition of the torsion scalar (2.8) we obtain

$$
T(r)=-2 K^{2} \frac{R^{\prime}}{R}\left(\frac{R^{\prime}}{R}+\frac{2 N^{\prime}}{N}\right),
$$

where primes denote derivatives with respect to $r$ and we denote $\kappa^{2}=\kappa_{4}^{2}=8 \pi G_{N}$. Also the field equations (2.18) become

$$
\begin{gathered}
\frac{1}{K^{2}}\left(\phi^{\prime 2}+\frac{2 V}{K^{2}}\right)+2\left(\frac{1}{\kappa^{2} K^{2}}-2 \xi \phi^{\prime 2}\right)\left(\frac{R^{\prime 2}}{R^{2}}+\frac{2 R^{\prime \prime}}{R}+2 \frac{R^{\prime}}{R} \frac{K^{\prime}}{K}-\frac{1}{K^{2} R^{2}}\right)-16 \xi \phi^{\prime} \frac{R^{\prime}}{R}\left(\frac{K^{\prime}}{K} \phi^{\prime}+\phi^{\prime \prime}\right)=0, \\
\frac{1}{K^{2}}\left(\phi^{\prime 2}-\frac{2 V}{K^{2}}\right)+2\left(2 \xi \phi^{\prime 2}-\frac{1}{\kappa^{2} K^{2}}\right)\left[\frac{R^{\prime}}{R}\left(\frac{R^{\prime}}{R}+\frac{2 N^{\prime}}{N}\right)-\frac{1}{K^{2} R^{2}}\right]+8 \xi \phi^{\prime 2} \frac{R^{\prime}}{R}\left(\frac{R^{\prime}}{R}+\frac{2 N^{\prime}}{N}\right)=0, \\
\frac{1}{K^{2}}\left(\phi^{\prime 2}+\frac{2 V}{K^{2}}\right)+2\left(\frac{1}{\kappa^{2} K^{2}}-2 \xi \phi^{\prime 2}\right)\left[\frac{N^{\prime}}{N}\left(\frac{R^{\prime}}{R}+\frac{K^{\prime}}{K}\right)+\frac{R^{\prime}}{R} \frac{K^{\prime}}{K}+\frac{N^{\prime \prime}}{N}+\frac{R^{\prime \prime}}{R}\right]-8 \xi \phi^{\prime}\left(\frac{R^{\prime}}{R}+\frac{N^{\prime}}{N}\right)\left(\frac{K^{\prime}}{K} \phi^{\prime}+\phi^{\prime \prime}\right)=0,
\end{gathered}
$$

$$
\phi^{\prime}\left(\frac{K^{\prime}}{K} \phi^{\prime}+\phi^{\prime \prime}\right)=0
$$

Equations (3.4)-(3.7) are accordingly the $(\lambda, \mu)=$ $t t, r r, \theta \theta, \theta r$ components of the system (2.18), while the other components vanish. The Klein-Gordon equation of the scalar field (2.20) becomes

$$
\left\{K N R^{2} \phi^{\prime}\left[1+4 \xi K^{2} \frac{R^{\prime}}{R}\left(\frac{R^{\prime}}{R}+\frac{2 N^{\prime}}{N}\right)\right]\right\}^{\prime}-\frac{N R^{2}}{K} \frac{d V}{d \phi}=0
$$

The off-diagonal equation (3.7) is a new feature of fouror higher-dimensional teleparallel gravity and it does not appear in the corresponding curvature-based equations of motion. It rises from the specific metric ansatz (3.1) and the vielbein (3.2) despite the fact that they are diagonal. Its appearance constrains the system of field equations, making them more difficult to be solved. Its physical significance is that it connects directly the scalar field to the metric function $K(r)$ of (3.1).

Equations (3.4)-(3.8) are invariant under $r$-reparametrizations, in the sense that when $r \rightarrow \tilde{r}(r)$ and $K \rightarrow K \frac{d \tilde{r}}{d r}$, $N \rightarrow N, R \rightarrow R, \quad \phi \rightarrow \phi$, the equations remain form invariant. This implies that one of these equations is expected to form the constraint of the theory, as will indeed be shown in the following. More specifically we have four functions, namely $N(r), K(r), \phi(r), V(r)$ [the function $R(r)$ is not considered in the enumeration because it corresponds to the choice of the radial gauge] and there are five equations of motion (3.4)-(3.8), but one of them is the constraint. So finally, we end up with four true equations for four unknowns. This is an interesting feature because the solution of the system for the diagonal vielbein will be basically unique without the need of any extra assumptions. On the contrary, in the curvature-based theories, for the same ansatz (3.1) the absence of the offdiagonal equation (3.7) provides three equations for the four unknowns. Therefore, one function has to be fixed arbitrarily, making the solutions quite sensitive to initial assumptions.

Assuming a nontrivial profile for the scalar field, Eq. (3.7) is integrated to give

$$
\phi^{\prime}=\frac{\nu}{K},
$$

where $\nu$ is an integration constant with dimensions of inverse length square. This is expected to characterize the charge of the scalar field. In Sec. IV we find an exact solution where the constant $\nu$ is related to the derivative coupling $\xi$ which determines the strength of the interaction of torsion to matter. However, in Sec. V, in the linearized solution found, these two parameters remain unrelated.

Using (3.9) Eqs. (3.4)-(3.6) and (3.8), along with the replacement of $\frac{R^{\prime \prime}}{R}+\frac{R^{\prime}}{R} \frac{K^{\prime}}{K}$ from (3.4) into (3.6), acquire the following form:

$\frac{2 R^{\prime \prime}}{R}+\frac{R^{\prime 2}}{R^{2}}+2 \frac{R^{\prime}}{R} \frac{K^{\prime}}{K}-\frac{1}{K^{2} R^{2}}+\frac{\kappa^{2}}{2\left(1-2 \xi \kappa^{2} \nu^{2}\right)} \frac{2 V+\nu^{2}}{K^{2}}=0$, 


$$
\begin{gathered}
\frac{1-6 \xi \kappa^{2} \nu^{2}}{\kappa^{2}} \frac{R^{\prime}}{R}\left(\frac{R^{\prime}}{R}+\frac{2 N^{\prime}}{N}\right) \\
+\left(V-\frac{\nu^{2}}{2}-\frac{1-2 \xi \kappa^{2} \nu^{2}}{\kappa^{2}} \frac{1}{R^{2}}\right) \frac{1}{K^{2}}=0 \\
\frac{N^{\prime \prime}}{N}+\frac{R^{\prime \prime}}{R}+\frac{N^{\prime}}{N}\left(\frac{R^{\prime}}{R}+\frac{K^{\prime}}{K}\right)+\frac{R^{\prime}}{R} \frac{K^{\prime}}{K} \\
+\frac{\kappa^{2}}{2\left(1-2 \xi \kappa^{2} \nu^{2}\right)} \frac{2 V+\nu^{2}}{K^{2}}=0 \\
\left\{N R^{2}\left[1+4 \xi K^{2} \frac{R^{\prime}}{R}\left(\frac{R^{\prime}}{R}+\frac{2 N^{\prime}}{N}\right)\right]\right\}^{\prime}-\frac{N R^{2}}{\nu K} \frac{d V}{d \phi}=0 .
\end{gathered}
$$

The above system of equations is quite complicated and we aim, after performing a series of transformations to obtain a single, decoupled, master equation from where we can get the behavior of the system. To do so, we consider as an independent argument the variable $\phi$ instead of the coordinate $r$ and we find for any $f(r)$ that $f^{\prime}=\frac{\nu}{K} \dot{f}$, $f^{\prime \prime}=\frac{\nu^{2}}{K^{2}}\left(\ddot{f}-\dot{f} \frac{\dot{K}}{K}\right)$, where $\dot{f}=\frac{d f}{d \phi}, \ddot{f}=\frac{d^{2} f}{d \phi^{2}}$. Then the system of field equations (3.10)-(3.13) becomes

$$
\begin{gathered}
\frac{\ddot{R}}{R}+\frac{\dot{R}^{2}}{2 R^{2}}-\frac{1}{2 \nu^{2} R^{2}}+\frac{\kappa^{2}\left(2 V+\nu^{2}\right)}{4 \nu^{2}\left(1-2 \xi \kappa^{2} \nu^{2}\right)}=0, \\
\frac{\dot{R}}{R}\left(\frac{\dot{R}}{R}+\frac{2 \dot{N}}{N}\right)+\frac{\kappa^{2}}{\nu^{2}\left(1-6 \xi \kappa^{2} \nu^{2}\right)} \\
\times\left(V-\frac{\nu^{2}}{2}-\frac{1-2 \xi \kappa^{2} \nu^{2}}{\kappa^{2}} \frac{1}{R^{2}}\right)=0, \\
\frac{\ddot{N}}{N}+\frac{\dot{N}}{N} \frac{\dot{R}}{R}-\frac{\dot{R}^{2}}{2 R^{2}}+\frac{1}{2 \nu^{2} R^{2}}+\frac{\kappa^{2}\left(2 V+\nu^{2}\right)}{4 \nu^{2}\left(1-2 \xi \kappa^{2} \nu^{2}\right)}=0, \\
\left\{N R^{2}\left[1+4 \xi \nu^{2} \frac{\dot{R}}{R}\left(\frac{\dot{R}}{R}+\frac{2 \dot{N}}{N}\right)\right]\right\}-\frac{N R^{2}}{\nu^{2}} \dot{V}=0 .
\end{gathered}
$$

Replacing (3.16) by the sum of (3.14) and (3.16) and defining

$$
\begin{gathered}
x=\ln R, \\
y=\frac{\dot{R}}{R}, \\
z=\frac{\left(R N^{2}\right)}{R N^{2}},
\end{gathered}
$$

Eqs. (3.14)-(3.17) become

$$
\begin{gathered}
\dot{y}+\frac{3}{2} y^{2}-\frac{e^{-2 x}}{2 \nu^{2}}+\frac{\kappa^{2}\left(2 V+\nu^{2}\right)}{4 \nu^{2}\left(1-2 \xi \kappa^{2} \nu^{2}\right)}=0 \\
y z=\frac{\kappa^{2}}{\nu^{2}\left(1-6 \xi \kappa^{2} \nu^{2}\right)}\left(\frac{1-2 \xi \kappa^{2} \nu^{2}}{\kappa^{2}} e^{-2 x}+\frac{\nu^{2}}{2}-V\right) \equiv F \\
\dot{z}+\frac{1}{2} z^{2}+\frac{e^{-2 x}}{2 \nu^{2}}+\frac{3 \kappa^{2}\left(2 V+\nu^{2}\right)}{4 \nu^{2}\left(1-2 \xi \kappa^{2} \nu^{2}\right)}=0 \\
(y z) \cdot-\frac{1}{4 \xi \nu^{4}} \dot{V}+\frac{1}{2}(3 y+z)\left(\frac{1}{4 \xi \nu^{2}}+y z\right)=0
\end{gathered}
$$

Thus, from (3.21), (3.23) we have

$$
\begin{aligned}
& (y z)+\frac{1}{2}\left[y z+\frac{\kappa^{2}\left(2 V+\nu^{2}\right)}{2 \nu^{2}\left(1-2 \xi \kappa^{2} \nu^{2}\right)}\right](3 y+z) \\
& +\frac{1}{2 \nu^{2}} e^{-2 x}(y-z)=0,
\end{aligned}
$$

while (3.22) gives

$\dot{V}=-2 \frac{1-2 \xi \kappa^{2} \nu^{2}}{\kappa^{2}} e^{-2 x} y-\frac{\nu^{2}\left(1-6 \xi \kappa^{2} \nu^{2}\right)}{\kappa^{2}}(y z)$.

Multiplying Eq. (3.26) by $-\frac{\kappa^{2}}{\nu^{2}\left(1-2 \xi \kappa^{2} \nu^{2}\right)}$ and adding this to Eq. (3.25) we get a new equation, in which when $V$ is replaced from (3.22), we obtain Eq. (3.24). This reveals that Eq. (3.22) is a constraint, and thus, Eq. (3.24) is redundant.

Note that the system of equations (3.4)-(3.6) for vanishing scalar field $\phi=0$ and a cosmological constant as the potential $V$ has the standard Schwarzschild-(A)dS solution. However, in the equivalent system (3.21)-(3.24) this limit has been lost, since setting $\nu=0$ in this system we get inconsistency.

Defining the new variables

$$
\begin{gathered}
Y \equiv y^{2} \\
Z \equiv z^{2},
\end{gathered}
$$

and using the field equations (3.21)-(3.23), we get a differential equation for $Y(x)$ as

$$
\begin{aligned}
2 \frac{d^{2} Y}{d x^{2}} & -\frac{1}{Y}\left(\frac{d Y}{d x}\right)^{2}+2\left(2-\frac{\eta \nu^{2}}{Y}\right) \frac{d Y}{d x}+3 Y+2 \eta \nu^{2} \\
-12 & \frac{\eta^{2}}{\tilde{\eta}^{2}} Y \frac{\frac{d Y}{d x}+3 Y-\frac{2}{3 \nu^{2}} e^{-2 x}}{\frac{d Y}{d x}+3 Y+2 \eta \nu^{2}}=0 .
\end{aligned}
$$

Equation (3.29) consists of the master equation which we were looking for, while the technical details for deriving this equation are given in Appendix A. The quantities $\eta, \tilde{\eta}$ are defined by Eqs. (A8), (A15). The strategy which we will 
follow is to find a solution of this equation and then use the other decoupled equations to specify the metric, the scalar field profile and the potential.

In particular, finding the function $Y$ from (3.29) allows us to use Eqs. (A4), (A16) in order to find $Z$ as

$$
Z=\frac{\tilde{\eta}^{2}}{4 \eta^{2} Y}\left(\frac{d Y}{d x}+3 Y+2 \eta \nu^{2}\right)^{2} .
$$

Moreover, combining Eqs. (A10), (A14), (A16), we find the potential $V$ as

$$
V=\frac{1}{2 \nu^{2} \eta} e^{-2 x}-\frac{\nu^{2}}{2}-\frac{1}{2 \eta}\left(\frac{d Y}{d x}+3 Y\right) .
$$

Additionally, from Eq. (A1) and the definitions (3.18), (3.19) we have

$$
\left(\frac{d x}{d \phi}\right)^{2}=Y(x)
$$

which can be integrated to provide $\phi(R)$. Then, we can use Eqs. (3.20), (A2) and (3.32) to obtain

$$
\left[\frac{d \ln \left(R N^{2}\right)}{d x}\right]^{2}=\frac{Z(x)}{Y(x)}
$$

the integration of which provides $N(R)$. Finally, using (3.9), (3.18) and (3.32) we can find

$$
K^{-2} d r^{2}=\frac{d R^{2}}{\nu^{2} R^{2} Y},
$$

and therefore the metric (3.1) acquires the form

$$
d s^{2}=-N^{2} d t^{2}+\frac{d R^{2}}{\nu^{2} R^{2} Y}+R^{2} d \Omega^{2} .
$$

Although we have managed to extract from the initial coupled system of field equations the master equation (3.29) plus the decoupled equations, the general solution of (3.29) is still difficult to be determined analytically. In the next two sections we will give two solutions of the theory and discuss their physical significance. The first one is a special exact solution of the field equations where the potential turns out to have a simple form. The second one is obtained from an asymptotic solution of the master equation (3.29).

\section{A NEW CLASS OF WORMHOLELIKE SOLUTIONS}

In this section we will find a special exact solution of the above system of equations, and in particular written in the form (A3)-(A5). Consider the potential

$$
V(x)=\alpha e^{-2 x}+\beta,
$$

where $\alpha$ and $\beta$ are constants. Then, from Eqs. (A6) and (A7) we obtain

$Y(x)=c_{1} e^{-3 x}+\left(\frac{1}{\nu^{2}}-2 \eta \alpha\right) e^{-2 x}-\frac{\eta}{3}\left(\nu^{2}+2 \beta\right)$,

$Z(x)=c_{2} e^{-x}-\left(\frac{1}{\nu^{2}}-6 \eta \alpha\right) e^{-2 x}-3 \eta\left(\nu^{2}+2 \beta\right)$,

where $c_{1}, c_{2}$ are integration constants. Choosing $c_{1}=c_{2}=0$ we find

$$
\begin{aligned}
& Y(x)=A e^{-2 x}-B, \\
& Z(x)=C e^{-2 x}-D,
\end{aligned}
$$

where

$$
\begin{aligned}
A & =\frac{1}{\nu^{2}}-2 \eta \alpha, \\
B & =\frac{\eta}{3}\left(\nu^{2}+2 \beta\right), \\
C & =6 \eta \alpha-\frac{1}{\nu^{2}}, \\
D & =9 B .
\end{aligned}
$$

Thus, inserting (4.4), (4.5) in (A4), we find that this equation is satisfied by choosing

case I: $\alpha=\frac{5}{8 \kappa^{2}}, \quad \beta=\nu^{2}=\frac{1}{8 \kappa^{2} \xi}$,

$$
\begin{array}{ll}
A=\frac{4 \kappa^{2} \xi}{3}, & B=\frac{2 \kappa^{2}}{3}, \\
C=12 \kappa^{2} \xi, & D=6 \kappa^{2},
\end{array}
$$

or

case II : $\alpha=\frac{1}{2 \kappa^{2}}, \quad \beta=\nu^{2}=\frac{1}{5 \kappa^{2} \xi}$,

$$
\begin{array}{ll}
A=\frac{5 \kappa^{2} \xi}{6}, & B=\frac{5 \kappa^{2}}{6}, \\
C=\frac{15 \kappa^{2} \xi}{2}, & D=\frac{15 \kappa^{2}}{2} .
\end{array}
$$

Since $Y, Z \geq 0$, it is implied that $A e^{-2 x}-B \geq 0$, $C e^{-2 x}-D \geq 0$, and therefore $A, C>0$, which requires $\xi>0$. Observe that in both cases, because the constant $\beta$ of the potential (4.1) is proportional to $1 / \xi$, it is scaled as $l^{-2}$ and therefore acts as a positive cosmological constant. Also note that the charge of the scalar field $\nu$ is proportional to $\sqrt{1 / \xi}$. Moreover, we deduce that $R \leq \sqrt{\frac{A}{B}}, \sqrt{\frac{C}{D}}$. This means that for the case (4.7) it is $R \leq \sqrt{2 \xi}$, while for the case (4.8) it is $R \leq \sqrt{\xi}$. 
Now, knowing the solution for $Y(x)$ from (4.4), we can use Eq. (3.32) to find the scalar field as

$$
\phi(R)=\phi_{1}+\frac{\epsilon_{1}}{\sqrt{B}} \arctan \left(\sqrt{\frac{A}{B} \frac{1}{R^{2}}-1}\right),
$$

where $\epsilon_{1}= \pm 1$ and $\phi_{1}$ is an integration constant. It proves to be convenient to redefine the field $\phi$, absorbing the integration constant $\phi_{1}$ and the index $\epsilon_{1}$. In particular, setting $\tilde{\phi}(R)=\epsilon_{1}\left[\phi(R)-\phi_{1}\right]$ we end up with

$$
\tilde{\phi}(R)=\frac{1}{\sqrt{B}} \arctan \left(\sqrt{\frac{A}{B} \frac{1}{R^{2}}-1}\right) .
$$

Thus, for case I it is

$$
\tilde{\phi}(R)=\sqrt{\frac{3}{2}} \frac{1}{\kappa} \arctan \left(\sqrt{\frac{2 \xi}{R^{2}}-1}\right),
$$

while for case II we have

$$
\tilde{\phi}(R)=\sqrt{\frac{6}{5}} \frac{1}{\kappa} \arctan \left(\sqrt{\frac{\xi}{R^{2}}-1}\right) .
$$

The potential $V$ can be now reconstructed by inverting (4.9), namely

$$
V(\phi)=\frac{\alpha B}{A} \tan ^{2}\left[\sqrt{B}\left(\phi-\phi_{1}\right)\right]+\frac{\alpha B}{A}+\beta,
$$

or

$$
V(\tilde{\phi})=\frac{\alpha B}{A} \tan ^{2}(\sqrt{B} \tilde{\phi})+\frac{\alpha B}{A}+\beta .
$$

Hence, for case I we have

$$
V(\tilde{\phi})=\frac{5}{16 \xi \kappa^{2}} \tan ^{2}\left(\sqrt{\frac{2}{3}} \kappa \tilde{\phi}\right)+\frac{7}{16 \xi \kappa^{2}},
$$

while for case II it is

$$
V(\tilde{\phi})=\frac{1}{2 \xi \kappa^{2}} \tan ^{2}\left(\sqrt{\frac{5}{6}} \kappa \tilde{\phi}\right)+\frac{7}{10 \xi \kappa^{2}} .
$$

The dependence of $V$ to $R$ for case $\mathrm{I}$ is

$$
V(R)=\frac{1}{8 \xi \kappa^{2}}+\frac{5}{8 \kappa^{2} R^{2}},
$$

while for case II

$$
V(R)=\frac{1}{5 \xi \kappa^{2}}+\frac{1}{2 \kappa^{2} R^{2}} .
$$

Note that the solutions for the scalar field and the potential have analogous forms with the corresponding solutions of the scalar field and potential of the MTZ solutions [relations (2.4) and (2.5) respectively]. However, while the cosmological constant $\Lambda=\kappa^{2} V(\tilde{\phi}=0)$ of the MTZ solution is an arbitrary parameter, here, the corresponding cosmological constant is given for case I by $\Lambda=$ $\frac{7}{16 \xi}$ and for case II by $\Lambda=\frac{7}{10 \xi}$. Thus, the gravity-scalar field equations have fixed this constant to depend on the coupling of torsion to the scalar field. Not only this, but also the integration constant $\nu$ of the scalar field is determined from the coupling $\xi$ through Eqs. (4.7), (4.8). This feature also occurs to the solutions of scalartensor theories, where usually the scalar charge of a local hairy black hole solution is related to the other scales of the theory like mass or charge, unless a symmetry is spontaneously broken, like conformal symmetry [38].

The lapse function can be calculated from Eq. (5.11). Using the parameter values from (4.6), (4.7), (4.8), we find

$$
\frac{d \ln \left(R N^{2}\right)}{d x}=3 \epsilon
$$

which has as a solution

$$
N(R)=\mathcal{C} R^{\frac{1}{2}(3 \epsilon-1)},
$$

with $\epsilon= \pm 1$ and $\mathcal{C}$ an integration constant. Therefore,

$N=\mathcal{C} R \quad$ for $\epsilon=1 \quad$ or $\quad N=\frac{\mathcal{C}}{R^{2}} \quad$ for $\epsilon=-1$.

Finally, inserting the above relations into (3.35), we find the metric

$d s^{2}=-\mathcal{C}^{2} R^{3 \epsilon-1} d t^{2}+\frac{d R^{2}}{\nu^{2}\left(A-B R^{2}\right)}+R^{2} d \Omega^{2}, \quad \epsilon= \pm 1$.

Thus, for case I it is

$$
d s^{2}=-\mathcal{C}^{2} R^{3 \epsilon-1} d t^{2}+\frac{12 \xi d R^{2}}{2 \xi-R^{2}}+R^{2} d \Omega^{2},
$$

while for case II we have

$$
d s^{2}=-\mathcal{C}^{2} R^{3 \epsilon-1} d t^{2}+\frac{6 \xi d R^{2}}{\xi-R^{2}}+R^{2} d \Omega^{2} .
$$

These metrics, together with $\tilde{\phi}(R)$ from (4.10) and $V(\tilde{\phi})$ from (4.14) form the exact spherically symmetric solutions that we have found. The solutions contain the two couplings $\kappa, \xi$ and one integration constant $\mathcal{C}$. Note that when the metric function in (4.23) or (4.24) has a singularity, i.e. at the maximum value $R^{2}=2 \xi$ or $R^{2}=\xi$, the accompanied scalar field $\tilde{\phi}$ vanishes, $\tilde{\phi}=0$. Moreover, at the origin $R=0$, the scalar field is still finite. Therefore, the scalar field is regular everywhere. However, the potential $V$ becomes infinite at the origin $R=0$, as is seen from (4.17), (4.18). Finally, the Ricci and Kretchmann 
scalars diverge at the origin but are finite elsewhere. For example, for the metric (4.24) and $\epsilon=1$ it is $R_{\mu \nu \kappa \lambda} R^{\mu \nu \kappa \lambda}=$ $\frac{2 R^{4}+2 \xi R^{2}+9 \xi^{2}}{3 \xi^{2} R^{4}}$.

Defining $b(R)=\nu^{2} B R^{3}+\left(1-\nu^{2} A\right) R$ the metric (4.22) can be written in the form

$$
d s^{2}=-\mathcal{C}^{2} R^{3 \epsilon-1} d t^{2}+\frac{d R^{2}}{1-b(R) / R}+R^{2} d \Omega^{2},
$$

where for case I it is $b(R)=\frac{1}{12 \xi} R^{3}+\frac{5}{6} R$, while for case II it is $b(R)=\frac{1}{6 \xi} R^{3}+\frac{5}{6} R$. The lapse function $N(R)$ is always regular, therefore, the metric (4.25) does not represent a black hole. It has the form of a wormhole $[63,64]$ with "throat" at $R=\sqrt{A / B}$. The difference is, however, that here the solution extends inside the throat up to the origin $R=0$, while in a usual wormhole the solution extends outside the throat up to the "mouth" or up to infinity. The behavior of this wormholelike solution is controlled by the length scale $\xi$ and this solution merits more investigation.

\section{SPHERICALLY SYMMETRIC SOLUTIONS AT LARGE DISTANCES}

In this section we will solve the master equation (3.29) perturbatively. We will assume that for $x \rightarrow \infty$, where $x$ is related to the radial distance as (3.18), the function $Y(x)$ has the expansion $Y(x)=Y_{0}+Y_{1}(x)+\ldots$, where $\left|Y_{1}(x)\right| \ll\left|Y_{0}\right|$. So, we will obtain large distance linearized solutions.

At zero order (3.29) gives

$$
Y_{0}=\frac{2 \eta \nu^{2}}{3(2 \sigma-1)}
$$

where we have introduced the constant

$$
\sigma \equiv \frac{\varepsilon \eta}{\tilde{\eta}}
$$

with $\varepsilon= \pm 1$. Since, due to (A1), it is $Y>0$, we have $Y_{0}>0$ and therefore $\eta(2 \sigma-1)>0$. Obviously the exponential factor in (3.29) is suppressed compared to the asymptotic value $Y_{0}$. To continue with the linearized correction $Y_{1}$ we assume additionally that $\left|Y_{1}(x)\right| \ll\left|Y_{0}+\frac{2 \eta \nu^{2}}{3}\right|$. The master equation (3.29) at first order, after substituting the zeroth order value (5.1), gives

$$
\begin{aligned}
& \frac{d^{2} Y_{1}}{d x^{2}}+\left[4(1-\sigma)+(1-2 \sigma) \frac{e^{-2 x}}{6 \eta \nu^{4}}\right] \frac{d Y_{1}}{d x} \\
& \quad+3(1-2 \sigma)\left[1+(1-2 \sigma) \frac{e^{-2 x}}{6 \eta \nu^{4}}\right] Y_{1}+\frac{2 \sigma e^{-2 x}}{3 \nu^{2}}=0
\end{aligned}
$$

Assuming further that $R \gg \frac{1}{2 \nu^{2}} \sqrt{\frac{|1-2 \sigma|}{6|\eta||1-\sigma|}}$, Eq. (5.3) becomes $\frac{d^{2} Y_{1}}{d x^{2}}+4(1-\sigma) \frac{d Y_{1}}{d x}+3(1-2 \sigma) Y_{1}+\frac{2 \sigma e^{-2 x}}{3 \nu^{2}}=0$.

The general solution of (5.4) is given by

$$
Y_{1}=c_{\lambda} e^{\lambda x}+c_{\mu} e^{\mu x}+\frac{2 \sigma}{3 \nu^{2}(1-2 \sigma)} e^{-2 x}
$$

where $c_{\lambda}, c_{\mu}$ are integration constants and

$$
\begin{aligned}
& \lambda=2(\sigma-1)-\sqrt{1-2 \sigma+4 \sigma^{2}}<0, \\
& \mu=2(\sigma-1)+\sqrt{1-2 \sigma+4 \sigma^{2}},
\end{aligned}
$$

with $\mu>\lambda$ being either positive or negative. Depending on the possible parameter values we have two cases:

(a) Case A: For $\sigma<\frac{1}{2}$ we have $\eta<0$. Then the only consistent case is for $\varepsilon=-1, \xi \kappa^{2} \nu^{2}>\frac{1}{2}$ (thus the coupling $\xi$ must be positive), and finally $\sigma<-\frac{3}{2}$. The values of $\lambda, \mu$ are $\lambda<-2,-3 / 2<\mu<0$, and therefore the total linearized solution becomes

$$
Y=\frac{2|\eta| \nu^{2}}{3(1-2 \sigma)}+c_{m} e^{-m x}+\frac{2 \sigma}{3 \nu^{2}(1-2 \sigma)} e^{-2 x}+c_{\ell} e^{-\ell x}
$$

with

$$
\begin{array}{r}
0<m=2(1-\sigma)-\sqrt{1-2 \sigma+4 \sigma^{2}}<\frac{3}{2} \\
\ell=2(1-\sigma)+\sqrt{1-2 \sigma+4 \sigma^{2}}>2,
\end{array}
$$

or in terms of the radial distance $R$

$$
Y=\frac{2|\eta| \nu^{2}}{3(1-2 \sigma)}+c_{m} R^{-m}+\frac{2 \sigma}{3 \nu^{2}(1-2 \sigma)} R^{-2}+c_{\ell} R^{-\ell},
$$

where $c_{\ell}, c_{m}$ are integration constants. According to the previous assumptions of linearization, the above solution is valid provided that $\mid c_{m} R^{2-m}+$ $\frac{2 \sigma}{3 \nu^{2}(1-2 \sigma)}+\frac{c_{\ell}}{R^{\ell-2}} \mid \ll \frac{2|\eta| \nu^{2}}{3(1-2 \sigma)} R^{2}, \frac{4|\eta| \nu^{2}|\sigma|}{3(1-2 \sigma)} R^{2}$. These inequalities mean that $R$ should be larger than some minimum distance, which decreases as $c_{m}$ decreases.

Using the above solution of the master equation and following the procedure described in Sec. III, the metric (3.35) is found to be

$$
\begin{aligned}
d s^{2}= & -N^{2} d t^{2} \\
& +\frac{d R^{2}}{\frac{2|\eta| \nu^{4}}{3(1-2 \sigma)} R^{2}+\mathrm{c}_{m} R^{2-m}+\frac{2 \sigma}{3(1-2 \sigma)}+\frac{\mathrm{c}_{\ell}}{R^{\ell-2}}}+R^{2} d \Omega^{2},
\end{aligned}
$$


where $\mathrm{c}_{m}=\nu^{2} c_{m}, \mathrm{c}_{\ell}=\nu^{2} c_{\ell}$ are redefined integration constants. The lapse metric function $N$ can be obtained from Eqs. (3.30) and (3.33) from which we obtain

$$
\frac{d \ln \left(R N^{2}\right)}{d x}=\frac{\zeta}{2 \sigma Y}\left(\frac{d Y}{d x}+3 Y+2 \eta \nu^{2}\right)
$$

where $\zeta= \pm 1$ is a sign symbol. Integrating Eq. (5.11) we extract the lapse function as

$$
N^{2}(R)=\frac{c}{R}\left[R^{3} Y(R) e^{2 \eta \nu^{2} J(R)}\right]^{\frac{\zeta}{2 \sigma}},
$$

where

$$
\begin{aligned}
J(R) & =\int \frac{d R}{R Y(R)} \\
& =\frac{\nu^{2}}{2} \int \frac{d u}{\frac{2|\eta| \nu^{4}}{3(1-2 \sigma)} u+\mathrm{c}_{m} u^{1-\frac{m}{2}}+\frac{2 \sigma}{3(1-2 \sigma)}+\left.\frac{\mathrm{c}_{\ell}}{u^{\frac{\ell}{2}-1}}\right|_{u=R^{2}}}
\end{aligned}
$$

and $c>0$ is an integration constant.

The potential can be determined after the insertion of the solution (5.9) into (3.31) to find

$$
\begin{aligned}
V= & \frac{(1+2 \sigma) \nu^{2}}{2(1-2 \sigma)}-\frac{3-m}{2 \eta \nu^{2}} \frac{\mathrm{c}_{m}}{R^{m}}+\frac{3-8 \sigma}{6(1-2 \sigma) \eta \nu^{2}} \frac{1}{R^{2}} \\
& +\frac{3-\ell}{2 \eta \nu^{2}} \frac{\mathrm{c}_{\ell}}{R^{\ell}} .
\end{aligned}
$$

Finally the scalar field configuration can be determined inserting the solution (5.9) into (3.32) to obtain

$\phi=\phi_{1}+\epsilon_{1} \nu \int \frac{d R}{\sqrt{\frac{2|\eta| \nu^{4}}{3(1-2 \sigma)} R^{2}+\mathrm{c}_{m} R^{2-m}+\frac{2 \sigma}{3(1-2 \sigma)}+\frac{\mathrm{c}_{\ell}}{R^{\ell-2}}}}$,

where $\epsilon_{1}$ is another \pm 1 sign and $\phi_{1}$ is an integration constant. Going back to the potential (5.14), we can find the next-to-leading order correction of $V$ as a function of the scalar field, namely

$V(\tilde{\phi}) \approx \frac{(1+2 \sigma) \nu^{2}}{2(1-2 \sigma)}-\frac{(3-m) \mathbf{c}_{m}}{2 \eta \nu^{2}} e^{-m \nu \sqrt{\frac{2||||}{3(1-2 \sigma)}} \tilde{\phi}}$,

where $\tilde{\phi}=\epsilon_{1}\left(\phi-\phi_{1}\right)$.

The integral (5.13) cannot be performed analytically, however, the leading asymptotic behavior is found to be $J(R) \approx \frac{3(1-2 \sigma)}{2|\eta| \nu^{2}} \ln R$, therefore, the leading asymptotic behavior of the lapse function is

$$
N^{2}(R) \approx c\left[\frac{2|\eta| \nu^{2}}{3(1-2 \sigma)}\right]^{\frac{\zeta}{2 \sigma}} \frac{1}{R^{1-3 \zeta}} .
$$

Of course, the exact integral (5.13) gives more information at even smaller distances. The asymptotic form of the metric (3.35) becomes

$$
\begin{aligned}
d s_{\infty}^{2}= & -c\left[\frac{2|\eta| \nu^{2}}{3(1-2 \sigma)}\right]^{\frac{\zeta}{2 \sigma}} \frac{1}{R^{1-3 \zeta}} d t^{2} \\
& +\frac{3(1-2 \sigma)}{2|\eta| \nu^{4}} \frac{d R^{2}}{R^{2}}+R^{2} d \Omega^{2} .
\end{aligned}
$$

Therefore, for the branch with $\zeta=1$, rescaling the time $t$ to $\tilde{t}=\frac{\sqrt{c}}{|\nu|}\left[\frac{2|\eta| \nu^{2}}{3(1-2 \sigma)}\right]^{\frac{\zeta}{6}}-\frac{1}{2} t$, we find the asymptotic metric

$$
d s_{\infty}^{2}=-\frac{2|\eta| \nu^{4}}{3(1-2 \sigma)} R^{2} d \tilde{t}^{2}+\frac{3(1-2 \sigma)}{2|\eta| \nu^{4}} \frac{d R^{2}}{R^{2}}+R^{2} d \Omega^{2} .
$$

The resulted metric (5.19) indicates that the space is asymptotically AdS and some interesting features appear. The length scale introduced here is given by $l_{\text {eff }}^{-2}=\frac{2|\eta| \nu^{4}}{3(1-2 \sigma)}$ and the corresponding effective cosmological constant is $\Lambda_{\text {eff }}=-3 l_{\text {eff }}^{-2}$. On the other hand, the potential $V$ of the solution asymptotically has a different negative constant given by Eq. (5.14) for $R \rightarrow \infty$. Therefore, the constant coming from the potential of the action and the effective cosmological constant of the asymptotically AdS spacetime can have different values, and this is due to the fact that the scalar field acquires a nontrivial profile at infinity. In this way it introduces another energy scale asymptotically. Note that the ratio of $\Lambda_{\text {eff }}$ and the constant appearing in the potential is proportional to $\xi \kappa^{2} \nu^{2}$, therefore, if $\nu^{2} \sim 1 / \kappa^{2} \xi$ as it happens in the exact solution we found, the two constants are of the same order. This behavior is different from the exact MTZ solution where the potential asymptotically becomes a cosmological constant which is the same with the cosmological constant of the asymptotically $\operatorname{AdS}$ spacetime seen in Eqs. (2.2), (2.3). Hence, in our case, the presence of the scalar field and its coupling to torsion modifies the asymptotic form of the spacetime.

In the linearized large distance solutions we discuss in this section the length scales, defined by the cosmological constants discussed above, depend on two quantities, the derivative coupling constant $\xi$ and the integration constant $\nu$ which expresses the scalar charge. Thus, contrary to the previous wormhole solution, in this case the two constants are not interrelated and this is explained since the linearized solution found is a general asymptotic solution with the correct number of integration constants. Note also 
that the derivative coupling constant $\xi$ enters the solution only through the specific combination $\xi \kappa^{2} \nu^{2}$, while the integration constant $\nu$ appears also independently. This combination is a remnant of the interaction term $T g^{\mu \nu} \partial_{\mu} \phi \partial_{\nu} \phi$ in the initial Lagrangian. Therefore, the value of $\xi$ is not significant on its own, but only in combination with the integration constant $\nu$ of the scalar field.

We note here that the aforementioned AdS behavior, as opposed to the dS behavior, creates an (extra) attraction force at local level at large distances, which may be useful at galactic or cluster of galaxies scales, and in particular can offer a novel mechanism for their stability. Also in the metric (5.10) the term $R^{2-m}$ expresses another large distance scale where gravity is modified, and for instance, in the case where $m=1$ this term becomes proportional to $R$. Then in [65] it was claimed that in Weyl gravity a linear term $R$ can successfully explain the galactic rotation curves without the need to add dark matter.

(b) Case B: For $\sigma>\frac{1}{2}$ we have $\eta>0$. Then the only consistent case is for $\varepsilon=-1, \frac{1}{4}<\xi \kappa^{2} \nu^{2}<\frac{1}{2}$ (thus the coupling $\xi$ must be positive) and $\eta>\frac{\kappa^{2}}{\nu^{2}}$. The values of $\lambda, \mu$ are $\mu>0,-2<\lambda<-3 / 2$, and thus it should be $c_{\mu}=0$ otherwise $Y_{1}$ keeps increasing at large distances and the condition $\left|Y_{1}\right| \ll\left|Y_{0}\right|$ is not satisfied. Therefore, for $\sigma>\frac{1}{2}$ the solution becomes

$Y=\frac{2 \eta \nu^{2}}{3(2 \sigma-1)}+c_{\ell} e^{-\ell x}-\frac{2 \sigma}{3 \nu^{2}(2 \sigma-1)} e^{-2 x}$

with

$$
\frac{3}{2}<\ell=2(1-\sigma)+\sqrt{1-2 \sigma+4 \sigma^{2}}<2,
$$

or in terms of $R$

$Y=\frac{2 \eta \nu^{2}}{3(2 \sigma-1)}+c_{\ell} R^{-\ell}-\frac{2 \sigma}{3 \nu^{2}(2 \sigma-1)} R^{-2}$,

where $c_{\ell}$ is an integration constant. The metric (3.35) takes the form

$d s^{2}=-N^{2} d t^{2}+\frac{d R^{2}}{\frac{2 \eta \nu^{4}}{3(2 \sigma-1)} R^{2}+\mathrm{c}_{\ell} R^{2-\ell}-\frac{2 \sigma}{3(2 \sigma-1)}}+R^{2} d \Omega^{2}$,

where $\mathrm{c}_{\ell}=\nu^{2} c_{\ell}$ is a redefined integration constant. Following the same procedure as in case A we find similar forms for the lapse function and the scalar field as in case A, while the form of the potential is found to be

$$
\begin{aligned}
V= & \frac{(1+2 \sigma) \nu^{2}}{2(1-2 \sigma)}-\frac{3-m}{2 \eta \nu^{2}} \frac{\mathrm{c}_{m}}{R^{m}}+\frac{3-8 \sigma}{6(1-2 \sigma) \eta \nu^{2}} \frac{1}{R^{2}} \\
& +\frac{3-\ell}{2 \eta \nu^{2}} \frac{\mathrm{c}_{\ell}}{R^{\ell}} .
\end{aligned}
$$

The physical significance of this solution is similar to the discussed case A solution.

\section{CONCLUSIONS}

In this work we investigated spherically symmetric solutions in scalar-torsion gravity theories. Using the teleparallel formulation of GR, which presents the torsion scalar field $T$, we allowed a derivative coupling of a scalar field $\phi$ to the torsion scalar. The resulted theory is a novel scalar-torsion theory different from the corresponding curvature-based gravity theories. It has the advantage of giving second-order field equations leading to a healthy theory without ghosts.

After extracting the general field equations, we applied them in the background of a spherically symmetric geometry. A novel feature of the arising coupled system of gravity-scalar field equations is the appearance of an offdiagonal equation, which couples the scalar field directly to a metric function. The appearance of this equation constrains further the system of the coupled field equations, making them difficult to be solved analytically. In spite of that, we managed to extract a single decoupled master equation, the solution of which allows us to find one by one all the other unknown functions.

Although the solution of the system of equations is unique for the metric components, the scalar field profile and the scalar field potential, we have found a specific form for the scalar potential which provides an exact solution of the field equations. This solution is special from the point of view of the number of integration constants, and as a result, both the integration constant of the scalar field and the asymptotic value of the potential depend on the derivative coupling constant. Interestingly, the obtained solution is a wormholelike one, however it extends inside the throat of the wormhole up to the origin, while in a usual wormhole the solution extends outside the throat up to the mouth or up to infinity. Moreover, the accompanied scalar field remains regular both at the origin and at the throat. Hence, this solution belongs to a new class of wormholelike solutions which can have interesting physical features.

Using the master equation, we obtained linearized solutions that are valid at large radial distances. Interestingly enough, we showed that at large distances the space is AdS, which implies that in the model at hand, at large distances we obtain a negative effective cosmological constant. However, the asymptotic form of the potential provides a different negative constant. This is a new feature, not present for example in the MTZ solution, and is due to the derivative coupling along with the nonvanishing 
asymptotic scalar field. Hence, in our case, the presence of the scalar field and its coupling to torsion modifies the asymptotic form of the spacetime. In these solutions the derivative coupling constant and the integration constant of the scalar field are not interrelated, contrary to the previous wormhole solution, and this is due to the fact that the solutions are general from the point of view of the number of integration constants.

The fact that the derivative coupling creates an anti-de Sitter space in the present solution, instead of a de Sitter one, gives an attractive term around a spherical object, which might have interesting implications on structure formation and cosmology. To the same direction may act the fact that apart from the above behavior, the radial metric potential acquires another contribution at smaller distances that can be close to linear in distance, and thus playing the role of an effective dark matter component.

In summary, as we observe, the derivative coupling in the framework of teleparallel gravity brings novel features that cannot be obtained in the usual curvature nonminimal couplings. However, a crucial test for the scalar-torsion theories to give distinct features from the scalar-tensor theories of GR is to find exact solutions representing a central object, like a black hole with scalar hair. Before considering this theory as a candidate for the description of nature it has to be shown that it is viable phenomenologically and the model parameters have to be determined.

\section{ACKNOWLEDGMENTS}

The authors wish to thank V. Cardoso and F. Lobo for the useful discussions. The research of ENS is implemented within the framework of the Action "Supporting Postdoctoral Researchers" of the Operational Program "Education and Lifelong Learning" (Actions Beneficiary: General Secretariat for Research and Technology), and is cofinanced by the European Social Fund (ESF) and the Greek State.

\section{APPENDIX: CALCULATION OF THE MASTER EQUATION}

In this appendix we will give the technical details for obtaining the master equation (3.29). Defining the variables

$$
\begin{aligned}
& Y \equiv y^{2}, \\
& Z \equiv z^{2},
\end{aligned}
$$

we find that $\dot{y}=\frac{1}{2} \frac{d Y}{d x}, \dot{z}=\frac{1}{2} \frac{d Z}{d x}$ and therefore Eqs. (3.21)(3.23) become

$$
\begin{gathered}
\frac{d Y}{d x}+3 Y-\frac{e^{-2 x}}{\nu^{2}}+\frac{\kappa^{2}\left(2 V+\nu^{2}\right)}{2 \nu^{2}\left(1-2 \xi \kappa^{2} \nu^{2}\right)}=0, \\
Y Z=F^{2},
\end{gathered}
$$

$$
\frac{d Z}{d x}+Z-\frac{e^{-2 x}}{\nu^{2}}+\frac{3 \kappa^{2}\left(2 V+\nu^{2}\right)}{2 \nu^{2}\left(1-2 \xi \kappa^{2} \nu^{2}\right)}=0 .
$$

Equations (A3), (A5) are linear differential equations whose solutions are

$$
\begin{aligned}
& Y(x)=e^{-3 x}\left(c_{1}+\frac{e^{x}}{\nu^{2}}-\frac{\eta}{3} \nu^{2} e^{3 x}-2 \eta \int V e^{3 x} d x\right), \\
& Z(x)=e^{-x}\left(c_{2}-\frac{e^{-x}}{\nu^{2}}-3 \eta \nu^{2} e^{x}-6 \eta \int V e^{x} d x\right),
\end{aligned}
$$

where $c_{1}, c_{2}$ are integration constants and we have defined the constant

$$
\eta \equiv \frac{\kappa^{2}}{2 \nu^{2}\left(1-2 \xi \kappa^{2} \nu^{2}\right)}
$$

Finally, inserting (A6), (A7) into (A4) we obtain an integrodifferential equation for the potential $V$, considered as a function of $x$, i.e. $V=V(x)$. Therefore, if the potential $V(x)$ was given, in general the system would be inconsistent, and the reason behind this is the extra off-diagonal equation (3.7). However, if the potential $V(x)$ satisfies this integrodifferential equation the system is consistent.

Let us transform the aforementioned integrodifferential equation into a differential equation. Defining the function

$$
h(x)=\int V e^{3 x} d x
$$

we immediately obtain

$$
V=e^{-3 x} \frac{d h}{d x}
$$

while $\quad \int V e^{x} d x=\int e^{-2 x} \frac{d h}{d x} d x=e^{-2 x} h+2 \int e^{-2 x} h d x$. Using $h(x)$, Eqs. (A6) and (A7) gives

$$
\begin{aligned}
Y(x) & =e^{-3 x}\left(c_{1}+\frac{e^{x}}{\nu^{2}}-\frac{\eta}{3} \nu^{2} e^{3 x}-2 \eta h\right), \\
Z(x)= & e^{-x}\left(c_{2}-\frac{e^{-x}}{\nu^{2}}-3 \eta \nu^{2} e^{x}-6 \eta e^{-2 x} h\right. \\
& \left.-12 \eta \int e^{-2 x} h d x\right)
\end{aligned}
$$

Thus, replacing $Z$ from (A4) in (A12) and differentiating we get

$$
\frac{d}{d x}\left(e^{x} \frac{F^{2}}{Y}\right)=\frac{e^{-x}}{\nu^{2}}-3 \eta \nu^{2} e^{x}-6 \eta e^{-2 x} \frac{d h}{d x} .
$$

Additionally, from (3.22) we deduce that 


$$
F=\tilde{\eta}\left(\frac{1}{2 \nu^{2} \eta} e^{-2 x}+\frac{\nu^{2}}{2}-e^{-3 x} \frac{d h}{d x}\right),
$$

where we have introduced the constant

$$
\tilde{\eta} \equiv \frac{\kappa^{2}}{\nu^{2}\left(1-6 \xi \kappa^{2} \nu^{2}\right)}
$$

Therefore, from (A11), (A14), the function $F$ can be expressed as

$$
F=\frac{\tilde{\eta}}{2 \eta}\left(\frac{d Y}{d x}+3 Y+2 \eta \nu^{2}\right)
$$

while (A13) becomes

$$
\frac{d}{d x}\left(e^{x} \frac{F^{2}}{Y}\right)=3 e^{x} \frac{d Y}{d x}+9 e^{x} Y-\frac{2}{\nu^{2}} e^{-x} .
$$

Hence, given (A16), Eq. (A17) is a second-order autonomous equation for $Y(x)$ which is also written as

$2 F \frac{d F}{d x}-\left(\frac{F^{2}}{Y}+3 Y\right) \frac{d Y}{d x}+F^{2}-9 Y^{2}+\frac{2}{\nu^{2}} e^{-2 x} Y=0$.

Finally, using (A16) and (A18) we extract the differential equation for $Y(x)$ as

$$
\begin{gathered}
2 \frac{d^{2} Y}{d x^{2}}-\frac{1}{Y}\left(\frac{d Y}{d x}\right)^{2}+2\left(2-\frac{\eta \nu^{2}}{Y}\right) \frac{d Y}{d x}+3 Y+2 \eta \nu^{2} \\
-12 \frac{\eta^{2}}{\tilde{\eta}^{2}} Y \frac{\frac{d Y}{d x}+3 Y-\frac{2}{3 \nu^{2}} e^{-2 x}}{\frac{d Y}{d x}+3 Y+2 \eta \nu^{2}}=0 .
\end{gathered}
$$

In summary, we have managed to replace the complicated system of field equations (3.4)-(3.8) with just Eq. (A19) plus the decoupled equations.
[1] M. Ostrogradski, Memoires sur les equations differentielles relatives au probleme des isoperimetres, Mem. Acad. Imp. Sci. St. Petersburg 4, 385 (1850).

[2] C. M. Will, The confrontation between general relativity and experiment, Living Rev. Relativity 9, 3 (2006).

[3] A. Unzicker and T. Case, Translation of Einstein's attempt of a unified field theory with teleparallelism, arXiv:physics/ 0503046.

[4] C. Möller, Conservation laws and absolute parallelism in general relativity, Mat. Fys. Medd. K. Dan. Vidensk. Selsk 1, 3 (1961).

[5] C. Pellegrini and J. Plebanski, Tetrad fields and gravitational fields, Mat. Fys. Medd. K. Dan. Vidensk. Selsk 2, 1 (1963).

[6] K. Hayashi and T. Shirafuji, New general relativity, Phys. Rev. D 19, 3524 (1979); Addendum to "New general relativity", Phys. Rev. D 24, 3312 (1981).

[7] R. Aldrovandi and J. G. Pereira, Teleparallel Gravity: An Introduction (Springer, Dordrecht, Netherlands, 2013).

[8] H. I. Arcos and J. G. Pereira, Torsion gravity: A reappraisal, Int. J. Mod. Phys. D 13, 2193 (2004).

[9] J. W. Maluf, The teleparallel equivalent of general relativity, Ann. Phys. (Berlin) 525, 339 (2013).

[10] J. G. Pereira, Teleparallelism: A new insight into gravity, Handbook of Spacetime, edited by A. Ashtekar and V. Petcov (Springer, Berlin, 2013).

[11] G. Kofinas and E. N. Saridakis, Teleparallel equivalent of Gauss-Bonnet gravity and its modifications, Phys. Rev. D 90, 084044 (2014).

[12] T. Wang, Static solutions with spherical symmetry in $f(T)$ theories, Phys. Rev. D 84, 024042 (2011).

[13] R.-X. Miao, M. Li, and Y.-G. Miao, Violation of the first law of black hole thermodynamics in $f(T)$ gravity, J. Cosmol. Astropart. Phys. 11 (2011) 033.
[14] C. G. Boehmer, A. Mussa, and N. Tamanini, Existence of relativistic stars in $f(T)$ gravity, Classical Quantum Gravity 28, 245020 (2011).

[15] M. H. Daouda, M. E. Rodrigues, and M. J. S. Houndjo, New static solutions in $f(T)$ theory, Eur. Phys. J. C 71, 1817 (2011).

[16] R. Ferraro and F. Fiorini, Spherically symmetric static spacetimes in vacuum $f(T)$ gravity, Phys. Rev. D 84, 083518 (2011).

[17] P. A. Gonzalez, E. N. Saridakis, and Y. Vasquez, Circularly symmetric solutions in three-dimensional teleparallel, $f(T)$ and Maxwell- $f(T)$ gravity, J. High Energy Phys. 07 (2012) 053.

[18] S. Capozziello, P. A. Gonzalez, E. N. Saridakis, and Y. Vasquez, Exact charged black-hole solutions in $D$-dimensional $f(T)$ gravity: Torsion vs curvature analysis, J. High Energy Phys. 02 (2013) 039.

[19] K. Atazadeh and M. Mousavi, Vacuum spherically symmetric solutions in $f(T)$ gravity, Eur. Phys. J. C 72, 2016 (2012).

[20] G. G. L. Nashed, Spherically symmetric charged-dS solution in $f(T)$ gravity theories, Phys. Rev. D 88, 104034 (2013).

[21] A. Paliathanasis, S. Basilakos, E. N. Saridakis, S. Capozziello, K. Atazadeh, F. Darabi, and M. Tsamparlis, New Schwarzschild-like solutions in $f(T)$ gravity through Noether symmetries, Phys. Rev. D 89, 104042 (2014).

[22] R. Ferraro and F. Fiorini, Modified teleparallel gravity: Inflation without inflaton, Phys. Rev. D 75, 084031 (2007).

[23] R. Ferraro and F. Fiorini, On Born-Infeld gravity in Weitzenbock spacetime, Phys. Rev. D 78, 124019 (2008).

[24] E. V. Linder, Einstein's other gravity and the acceleration of the Universe, Phys. Rev. D 81, 127301 (2010). 
[25] S. H. Chen, J. B. Dent, S. Dutta, and E. N. Saridakis, Cosmological perturbations in $f(T)$ gravity, Phys. Rev. D 83, 023508 (2011)

[26] J. B. Dent, S. Dutta, and E. N. Saridakis, $f(T)$ gravity mimicking dynamical dark energy. Background and perturbation analysis, J. Cosmol. Astropart. Phys. 01 (2011) 009.

[27] K. Bamba, C. Q. Geng, C. C. Lee, and L. W. Luo, Equation of state for dark energy in $f(T)$ gravity, J. Cosmol. Astropart. Phys. 01 (2011) 021.

[28] S. Capozziello, V. F. Cardone, H. Farajollahi, and A. Ravanpak, Cosmography in $f(T)$-gravity, Phys. Rev. D 84, 043527 (2011).

[29] J. T. Li, C. C. Lee, and C. Q. Geng, Einstein static Universe in exponential $f(T)$ gravity, Eur. Phys. J. C 73, 2315 (2013).

[30] Y. C. Ong, K. Izumi, J. M. Nester, and P. Chen, Problems with propagation and time evolution in $f(T)$ gravity, Phys. Rev. D 88, 024019 (2013).

[31] G. Kofinas, G. Leon, and E. N. Saridakis, Dynamical behavior in $f\left(T, T_{G}\right)$ cosmology, Classical Quantum Gravity 31, 175011 (2014).

[32] G. Kofinas and E. N. Saridakis, Cosmological applications of $F\left(T, T_{G}\right)$ gravity, Phys. Rev. D 90, 084045 (2014).

[33] R. Ferraro and F. Fiorini, Remnant group of local Lorentz transformations in $f(T)$ theories, Phys. Rev. D 91, 064019 (2015).

[34] N. Bocharova, K. Bronnikov, and V. Melnikov, Vestn. Mosk. Univ., Ser. 3: Fiz., Astron. 6, 706 (1970); J. D. Bekenstein, Exact solutions of Einstein-conformal scalar equations, Ann. Phys. (N.Y.) 82, 535 (1974); Black holes with scalar charge, Ann. Phys. (N.Y.) 91, 75 (1975).

[35] K. A. Bronnikov and Y. N. Kireyev, Instability of black holes with scalar charge, Phys. Lett. 67A, 95 (1978).

[36] C. Martinez, R. Troncoso, and J. Zanelli, Exact black hole solution with a minimally coupled scalar field, Phys. Rev. D 70, 084035 (2004).

[37] C. Martinez, J. P. Staforelli, and R. Troncoso, Topological black holes dressed with a conformally coupled scalar field and electric charge, Phys. Rev. D 74, 044028 (2006).

[38] T. Kolyvaris, G. Koutsoumbas, E. Papantonopoulos, and G. Siopsis, A new class of exact hairy black hole solutions, Gen. Relativ. Gravit. 43, 163 (2011).

[39] P. A. González, E. Papantonopoulos, J. Saavedra, and Y. Vásquez, Four-dimensional asymptotically AdS black holes with scalar hair, J. High Energy Phys. 12 (2013) 021.

[40] P. A. González, E. Papantonopoulos, J. Saavedra, and Y. Vásquez, Extremal hairy black holes, J. High Energy Phys. 11 (2014) 011.

[41] V. Sahni and S. Habib, Does Inflationary Particle Production Suggest $\Omega_{m}$ Less than 1?, Phys. Rev. Lett. 81, 1766 (1998).

[42] J.-P. Uzan, Cosmological scaling solutions of nonminimally coupled scalar fields, Phys. Rev. D 59, 123510 (1999).

[43] N. Bartolo and M. Pietroni, Scalar tensor gravity and quintessence, Phys. Rev. D 61, 023518 (1999).

[44] L. Amendola, Cosmology with nonminimal derivative couplings, Phys. Lett. B 301, 175 (1993).

[45] S. V. Sushkov, Exact cosmological solutions with nonminimal derivative coupling, Phys. Rev. D 80, 103505 (2009).
[46] G. W. Horndeski, Second-order scalar-tensor field equations in a four-dimensional space, Int. J. Theor. Phys. 10, 363 (1974).

[47] C. Gao, When scalar field is kinetically coupled to the Einstein tensor, J. Cosmol. Astropart. Phys. 06 (2010) 023.

[48] S. Sushkov, Realistic cosmological scenario with nonminimal kinetic coupling, Phys. Rev. D 85, 123520 (2012).

[49] E. N. Saridakis and S. V. Sushkov, Quintessence and phantom cosmology with non-minimal derivative coupling, Phys. Rev. D 81, 083510 (2010).

[50] T. Kolyvaris, G. Koutsoumbas, E. Papantonopoulos, and G. Siopsis, Scalar hair from a derivative coupling of a scalar field to the Einstein tensor, Classical Quantum Gravity 29, 205011 (2012).

[51] M. Rinaldi, Black holes with nonminimal derivative coupling, Phys. Rev. D 86, 084048 (2012).

[52] T. Kolyvaris, G. Koutsoumbas, E. Papantonopoulos, and G. Siopsis, Phase transition to a hairy black hole in asymptotically flat spacetime, J. High Energy Phys. 11 (2013) 133.

[53] S. M. M. Rasouli, A.H. Ziaie, J. Marto, and P. V. Moniz, Gravitational collapse of a homogeneous scalar field in deformed phase space, Phys. Rev. D 89, 044028 (2014).

[54] A. Anabalon, A. Cisterna, and J. Oliva, Asymptotically locally AdS and flat black holes in Horndeski theory, Phys. Rev. D 89, 084050 (2014).

[55] A. Cisterna and C. Erices, Asymptotically locally AdS and flat black holes in the presence of an electric field in the Horndeski scenario, Phys. Rev. D 89, 084038 (2014).

[56] C. Charmousis, T. Kolyvaris, E. Papantonopoulos, and M. Tsoukalas, Black holes in bi-scalar extensions of Horndeski theories, J. High Energy Phys. 07 (2014) 085.

[57] C.-Q. Geng, C.-C. Lee, E. N. Saridakis, and Y.-P. Wu, "Teleparallel" dark energy, Phys. Lett. B 704, 384 (2011).

[58] C.-Q. Geng, C.-C. Lee, and E. N. Saridakis, Observational constraints on teleparallel dark energy, J. Cosmol. Astropart. Phys. 01 (2012) 002.

[59] C. Xu, E. N. Saridakis, and G. Leon, Phase-space analysis of teleparallel dark energy, J. Cosmol. Astropart. Phys. 07 (2012) 005.

[60] G. Otalora, Scaling attractors in interacting teleparallel dark energy, J. Cosmol. Astropart. Phys. 07 (2013) 044.

[61] M. A. Skugoreva, E. N. Saridakis, and A. V. Toporensky, Dynamical features of scalar-torsion theories, Phys. Rev. D 91, 044023 (2015).

[62] P. A. González, J. Saavedra, and Y. Vásquez, Threedimensional hairy black holes in teleparallel gravity, arXiv:1411.2193.

[63] M. Visser, Lorentzian Wormholes: From Einstein to Hawking, AIP Series in Computational and Applied Mathematical Physics (American Institute of Physics, Woodbury, NY, 1995).

[64] J. P. S. Lemos, F. S. N. Lobo, and S. Quinet de Oliveira, Morris-Thorne wormholes with a cosmological constant, Phys. Rev. D 68, 064004 (2003).

[65] P. D. Mannheim and D. Kazanas, Exact vacuum solution to conformal Weyl gravity and galactic rotation curves, Astrophys. J. 342, 635 (1989). 